Journal of Education and Science (ISSN 1812-125X), Vol: 30, No: 3, 2021 (167-177)

Special Issue for Proceeding of $3^{\text {rd }}$ National (1 $1^{\text {st }}$ international conference of biology) (ICBSUM 2021) 5, 6 July

College of Education for Pure Science, University of Mosul, Mosul, Iraq.

\title{
Effect of four inert dusts on some biological aspects of Khapra beetle Trogoderma granarium Everts (Coleoptera: Dermestidae)
}

\author{
Ali A. Ali Almimari ${ }^{1 *}$, Ibrahim Kh. Ibrahim Alhadidy ${ }^{2}$ \\ ${ }^{1 *, 2}$ Department of Biology, Education College for Pure Science, University of Mosul, Mosul, Iraq \\ E-mail: ${ }^{1 *}$ ali.esp46@ student.uomosul.edu.iq, ${ }^{2}$ dr.ibrahimkhaleel@ uomosul.edu.iq
}

(Received April 25, 2021; Accepted June 08, 2021; Available online August 28, 2021)

DOI: 10.33899/edusj.2021.168645, (c) 2021, College of Education for Pure Science, University of Mosul.

This is an open access article under the CC BY 4.0 license (http://creativecommons.org/licenses/by/4.0/).

\begin{abstract}
In this study four types of inert dusts were mixed with wheat grains at three concentrations 5, 7 and $9 \mathrm{gm} / \mathrm{kgm}$ of wheat to estimate their effect on some biological aspects of Khapra beetle Trogoderma granarium Everts. These inert dusts are Chalky limestone, Bituminous limestone, Sand and Celite.

The results indicated that these inert dusts affected on some biological aspects of this insect, present results showed that the inert dusts caused death to larvae and pupae during its growth and development, and this led to reduce the number of insects, mortality rate of larvae increased to 46.64, 55.56, 27.78 and 87.78\% for Chalky limestone, Bituminous limestone, Sand and Celite, respectively, as compared to $0.0 \%$ for the control. Also mortality rate of pupae increased to $15.02,23.89,3.69$ and $27.77 \%$, respectively, as compared to $0.0 \%$ for the control. This inert dusts led to significant increase of the larval period whereas were $30.05,31.69,27.41$ and 33.85 day, respectively, as compared to 24.07 day for the control. Also inert dusts led to significant decrease of the emerging adults number whereas were 4.56, 3.56, 6.44 and 2.67 insect, respectively, as compared to 10.0 insect for the control, also inert dusts led to significant decrease of the laid eggs number whereas were 16.56, 16.44, 20.89 and 17.67 egg/ female, respectively, as compared to $34.67 \mathrm{egg} /$ female for the control.
\end{abstract}

Keyword: Inert dusts, Chalky limestone, Bituminous limestone, Sand, Celite, Khapra beetle.

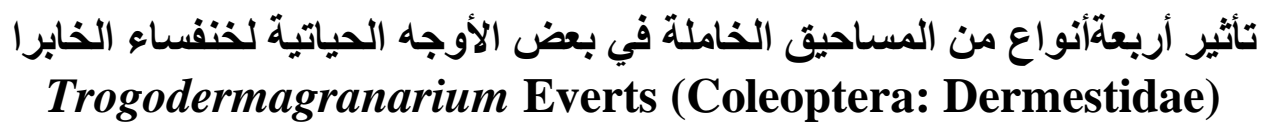

$$
\begin{aligned}
& \text { علي عبدالله علي المعماري1"، إبراهيم خليل إبراهيم الحديدي2 } \\
& \text { 1*2، قسم علوم الحباة، كلية التربية للعلوم الصرفة، جامعة الموصل، الموصل، العراق }
\end{aligned}
$$

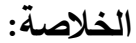

اجريت هذه الدراسة لاختبار فاعلية أربعة أنواع من المساحيق الخاملة وهي الحجر الجيري الطباشيري Chalky limestone،

الحجر الجيري القيري Bituminous limestone، الرمل Sand و السيلايت Celite عند خلطها مع حبوب الحنطة عند التراكيز 5،

7 و 9 غم/ كغم في بعض الأوجه الحياتية لخنفساء الخابرا Trogoderma granarium Everts. 
Journal of Education and Science (ISSN 1812-125X), Vol: 30, No: 3, 2021 (167-177)

Special Issue for Proceeding of $3^{\text {rd }}$ National (1 $1^{\text {st }}$ international conference of biology) (ICBSUM 2021) 5, 6 July

College of Education for Pure Science, University of Mosul, Mosul, Iraq.

بينت النتائج بان تلك المساحيق الخاملة قد اثرت على بعض الاوجه الحياتية لهذه الحشرة، اذ اظهرت النتائج ان المساحيقالخاملة المستخدمة سببت هلاك في اليرقات والعذارى اثناء نموها وتطورها مما ادى الى انخفاض عدد كاملات الحشرة الخارجة، اذ ارتفعت النسبة المئوية لليرقات الميتة مقارنة بالمجموعة الضابطة فكانت 46.67، 55.56، 27.78 و 87.78\% لكل من الحجر الجيري الطباشيري، الحجر الجيري القيري، الرمل و السيلايت، على التوالي، مقارنة بـ 0.0\% في المجموعة الضابطة، كما ارتفعت النسبة المئوية للعذارى الميتة في المعاملات مقارنة بالمجموعة الضابطة فكانت 15.02، 23.89، 3.69 و 27.77\%، على التوالي، مقارنة بـ 0.0\% في المجموعة الضابطة. ونتج عن المعاملة بالمساحيق الخاملة المستخدمة اطالة ملحوظة في مدة الطور اليرقي فكانت 30.05، 31.69، 27.41 و 33.85 يوماً، على التوالي، مقارنة بـ 24.07 يوم في المجموعة الضابطة. كما انخفضت بشكل معنوي عدد الحشرات الخارجة نتيجة المعاملة بالمساحيق الخاملة فكانت 4.56، 3.56، 6.44 و 2.67 حشرة، على التوالي، مقارنة بـ 10 حشرات في المجموعة الضابطة، كما انخفضت بشكل معنوي عدد البيض الموضوع لكل انثى نتيجة المعاملة بالمساحيق الخاملة فكانت 16.56، 16.44، 20.89 و17.67 بيضة/ انثى، على التوالي، مقارنة بـ 34.67 بيضة/ انثى المجموعة الضابطة. الكلمات المفتاحية : مساحيق خاملة، الحجر الجيري الطباشيري، الحجر الجيري القيري، الرمل، السيلايت، خنفساء الخابرا

1

يعد القهح .Triticum aestivum L من أهم محاصيل الحبوب ذات الأهمية العالمية، وان تحسين محصول الحبوب والسمات التي تساهم في زيادة كمية إنتاج القهح أمر ضروري ومهم للأمن الغذائي نتيجة الزيادة السكانية المستمرة[1]، ويحتل القـح المرتبة الأولى في العراق والعالم من حيث الأراضي الصالحة للزراعة وحجم الإنتاج والعائد المادي. يؤمن القمح للإنسان حوالي25\% من السعرات الحرارية والبروتينات لذلك فهو المصدر الرئيسي للغذاء لأكثر من مليار ونصف المليار نسمة في 40 دوله يسكنها 35\% من سكان العالم [2]، كما يعتبر محصول القمح من أهم محاصيل الحبوب في العراق اذ يشكل انتاج القمح في المنطقة الثمالية 69.1\% من إجمالي الإنتاج العراقي، وتحتل محافظة نينوى المرتبة الأولى من حيث الإنتاج بنسبة 35.5\% وذلك لوجود الأراضي الخصبة والظروف المناخية الملائمة والخبرات الزراعية المتراكمة، ويساهم هذا المحصول في توفير دخل للمزارعين العراقيين وخفض الواردات من الخارج، كما يساهم بنسبة كبيرة في توفير الغذاء الأساسي للإنسان لاحتوائه على نسبة عالية من الكربوهيدرات والبروتينات والفيتامينات[3]،[4]، وقد بين [5] ان التركيب الكيميائي لحبة الحنطة هي 67-71\% نشاء، 10-15\% بروتين، 8-10\% سكريات معقدة، 2-4\% سيليلوز ،

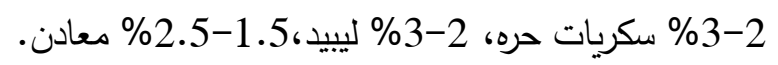

تواجه الحبوب العديد من المشاكل من مرحلة الحصاد والتخزين إلى التسويق والاستهلاك من الناحية الاقتصادية والصحية، وعانى العالم ولايزال يعاني من أضرار الآفات نتيجة هجوم العديد من الحشرات التي تسبب أضراراً جسيمة سواء من حيث الكمية أو النوعية في الحبوب أثناء التخزين[6]، وقد أثشار[7] إلى تعرض الحبوب المخزونة ومنتجاتها للعديد من الآفات التي تسبب خسائر اقتصادية كبيرة تقدر بحوالي10\%-40\% من الحبوب المخزونة في العالم، ويبلغ فقدان الوزن من حبوب القهح المخزونة 60\% بعد خمسة عشر أسبوعاً فقط من التخزين[8]، ويقدر فقدان الحبوب الناجم من الآفات الحشرية أثناء التخزين بنحوبة الحبوب في بعض مناطق افريقيا وارتيريا واغندا وبنحو 80\% في دول العالم الثالث[9]. يعتبر دقيق القمح أهم الأغذية للإنسان والأكثر انتشاراً محلياً وعالمياً، ولكن في العديد من الدول الآسيوية والإفريقية يتم تخزين كميات كبيرة منه في المستودعات المعرضه للهواء في 
Journal of Education and Science (ISSN 1812-125X), Vol: 30, No: 3, 2021 (167-177)

Special Issue for Proceeding of $3^{\text {rd }}$ National (1st international conference of biology) (ICBSUM 2021) 5, 6 July

College of Education for Pure Science, University of Mosul, Mosul, Iraq.

ظل ظروف التخزين غير الملائمة حيث يكون الدقيق عرضه للعدوى بالعوامل البيولوجية والسمية والإصابة بالآفات ومن بينها خنفساء T. Trogoderma granarium Everts مما يؤثر على مدى ملائمته للاستهلاك البشري[10]، حيث تعد خنفساء الخابرا granarium Everts في جميع أنحاء العالم، وهي بذلك تثكل تهديدا كبيرا للأمن الغذائي العالمي[11]. ان الاستخدام المفرط للمبيدات في حماية المحاصيل والمواد المخزونة من آفات الحبوب أدى إلى ظهور عدد من المشاكل الجانبية تمثلت في ظهور أجيال جديدة من الحشرات المقاومة

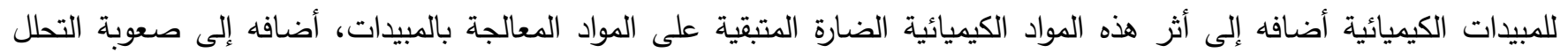
البطيء لهذه المبيدات في البيئة[12]، كما ان استخدام المبيدات الكيميائية للمحافظة على السلع الغذائية من الآفات الحشرية يثير القلق عالمياً لما لهذه المبيدات من تأثيرات سلبية على البيئة والصحة العامة، لذا تم الاتجاه إلى استخدام ما تحتويه النباتات من مواد باعتبارها

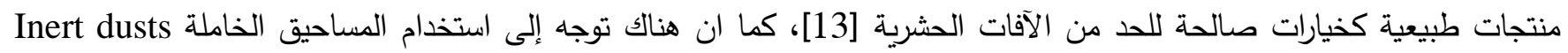
كإحدى الطرائق المهمة في المكافحة الفيزيائية للآفات الحشرية، حيث تتميز بسميتها المنخفضة للثدييات، وانخفاض سعرها، وسهولة تطبيقها، وحماية البذور المخزونة لفترة طويلة، من بين هذه المساحيق الكادلينت والسيلكا والترب الدياتومية, حيث أن مركبات السيلكاSilca، والرمل:Sand، والزيوليت، Zoeolet، والطين Clay هي أساس مكوناتها، هذه المساحيق أثثتت فعاليتها في مكافحة الحشرات حيث انها تخد البشرة وتمتص الماء والدهون مما يتسبب في فقدان الماء من جسم الحشرات فيؤذي ذلك إلى تصلب في جسم الحشرة وموتها، كما تؤثر هذه المساحيق على الجهاز التنفسي من خلا إغلاق فتحات التتفس مما يؤذي إلى اختتاق الآفات وموتها[14]،[15]،[16]، ولكون البذور المخزونة عرضه للتلف بسبب عدة عوامل بما في ذلك الإصابة بالآفات الحشرية وبالتي تسبب خسائر اقتصادية جسيمة إذا لم يتم إتباع الطرائق السليمة والوقائية، وبناءاً على ما ذكر فقد أجريت هذه الدراسة لاختبار ومقارنة كفاءة وفاعلية مساحيق أربعة أنواع من الصخور وهي: الحجر الجيري الطباشيري Chalky limestone، الحجر الجيري القيري:Bituminous limestone، الرمل Sand، والسيلايت Celite في بعض الأوجه الحياتية لخنفساء الخابرا Trogoderma granarium Everts

2. 20 - مواد وطرائق العمل

تم تربية مستعمرات خنفساء الخابرا Trogoderma granarium Everts من المستعمرات المتوفرة سابقاً من هذا النوع من الحشرات في مختبر بحوث الحشرات في كلية التربية للعلوم الصرفة، جامعة الموصل، منذ فترة زمنية طويلة في بيئة قياسية تتكون من95\%بذور حنطة بالإضافة إلى 5\% من مسحوق خميرة جافة في قناني زجاجية سعة 600 سم3 وغطيت فوهاتها بقماش الململ واحكم ربطها باستخدام أحزمة مطاطية ثم وضعت في الحاضنة عند درجة حرارة 32×0م، ورطوبة نسبية60 55\%،وتم التأكد من تشخيصها بالاعتماد على [17]. استخدمت في البحث حبوب حنطة صنف اباء99 سليمة خالية من الإصابة، تم الحصول عليها من دائرة فحص وتصديق البذور، فرع نينوى/وزارة الزراعة، وكانت بالمواصفات الآتية (المحتوى الرطوبي.6.5-7\%،النقاوة 90\%)،وتم تنظيف الحبوب يدوياً لإزالة المواد الغريبة وغربلتها بغربال ذو فتحات 2ملم، ثم وضعت الحبوب تحت التجميد لمدة 24 ساعة للقضاء على اية إصابة حشرية محتملة[18]. تم سحق الصخور المستخدمة في الدراسة باستخدام جهاز خاص لطحن الصخور في الورشة التابعة لقسم علوم الأرض/ كلية العلوم/ جامعة الموصل ثم نخلت المساحيق باستخدام منخل قياس 0.250)mesh 60 ملم) للحصول 
Journal of Education and Science (ISSN 1812-125X), Vol: 30, No: 3, 2021 (167-177)

Special Issue for Proceeding of $3^{\text {rd }}$ National (1 $1^{\text {st }}$ international conference of biology) (ICBSUM 2021) 5, 6 July

College of Education for Pure Science, University of Mosul, Mosul, Iraq.

على مساحيق ناعمة. تم اخذ عدد من الحشرات الكاملة ذكوراً واناثاً حديثة الخروج بعدما تم عزلها عندما كانت في طور العذراء من مستعمرات التربية وتركت تحت المراقبة اليومية بعد نقلها إلى اطباق بلاسيتكية حاوية على الحنطة كغذاء وتركت في الحاضنة لتضع البيض، وتم مراقبة البيض لحين الفقس وخروج يرقات العمر الأول[19]. تم نقل 10 يرقات من يرقات العمر الأولإلى اطباق بتري بلاستيكية ارتفاعها 1.5 سم وقطرها 8 سم تحتوي على 5 غم حبوب حنطة مخلوطة مع المساحيق الخاملة بتراكيز 5، 7، 9 غم مسحوق/ كغم حبوب حنطة، حفظت الاطباق في الحاضنة عند درجة حرارة 3210م، ورطوبة نسبية60 5 5\%، اعيدت التجربة اعلاه بواقع 3 مكررات لكل تركيز ولكل مسحوق فضلا عن معاملة المقارنة التي لم يضاف اليها المسحوق. تمت متابعة حياتية الحشرة وذلك بحساب مدة الطور اليرقي والعذري، نسبة الموت في الطور اليرقي والعذري، عدد الحشرات الكاملة الخارجة، تقدير عمر البالغات لزوج واحد فقط واستبعاد البقية، عدد البيض لكل انثى، مدة حضانة البيض والنسبة المئوية لفقس البيض.

تم تقدير نسبة الانبات للحبوب المعاملة بالمساحيق وفقا لقواعد منظمة فحص البذور الدولية [ISTA[20]، وذلك بعد فترة تخزين 6 اشهر، اذ اخذت 30 حبة حنطة من كل معاملة وبواقع 3 مكررات إضافة إلى معاملة المقارنة والتي تركت بدون اضافة المساحيق والتي تركت لمدة 6 اشهر ايضاً، وبعد فترة ال 6 اشهر عقدت الحنطة بمحلول هيبوكلورات الصوديوم بتركيز 1\% لمدة دقيقتين ثم غسلت الحنطة بالماء المقطر المعقم ومن ثم نقلت الى اطباق بتري حاوية على ورق ترشيح (نوع 604 Schleicher and Schuell الماني الصنع) ورطبت بقليل من الماء المقطر، وحظنت الاطباق على درجة حرارة 250م ثم فحصت بعد اسبوع وتم حساب نسبة انبات البذور حسب المعادلة التالية:-

$$
\text { النسبة المئوية للانبات= (عدد البذور النابتة؛ عدد البذور الكلي) × } 100 \text { [21]. }
$$

استعمل التصميم العشوائي الكامل العاملي Factorial Completely Randomized Design (FCRD) لكل التجارب وبعاملين، العامل الاول هو تراكيز المسحوق المختلفة والعامل الثاني هو نوع المسحوق[22]. ادخلت البيانات وحللت باستخدام البرنامج الاحصائي SPSS[23] تم استعمال تحليل التباين ANOVA) Analysis of variance) لحساب التباين وتاثير العوامل الداخلة في التجربة، ولمقارنة النتائج إحصائياً تم استعمال اختبار دنكن للمتوسطات المتعددة Duncan's multiple range test عند مستوى احتمال 0.05 لتحديد المعنوية بين المتغيرات.

3.

يبين جدول (1) تأثير المسـاحيق الخاملـة في حياتيـة خنفسـاء الخـابرا Trogoderma granarium، اذ أدت هذه المسـاحيق وبتراكيزها المختلفة إلى إطالة مدة الطور اليرقي مقارنة بالمجموعة الضـابطة وهذه الإطالة تفاوتت من مسحوق إلى أخر ومن تركيز إلى أخر فكانت أطول مدة للطور اليرقي 34.2 يوم عند التركيز 9غم مسحوق الحجر الجيري القيري/ كغم حنطة مقارنة بمدة الطور اليرقي في المجموعة الضـابطة حيث كانت 24.07 يوم، وهذا يتوافق مع ما توصل اليه [24] من ان مدة الطور اليرقي عموما طالت بفعل المساحيق الخاملة مقارنة بالمجموعة الضـابطة. وكانت مدة الطور اليرقي أقل تأثرا عند التركيز 5غم مسحوق الرمل/كغم حنطة حيث كانت 25.7 يوم أي بفارق 1.63 يوم مقارنة بالمجموعة الضابطة. كما لم تتكن اليرقات من اكمال الطور اليرقي عند التركيزين 7 و9 
Journal of Education and Science (ISSN 1812-125X), Vol: 30, No: 3, 2021 (167-177)

Special Issue for Proceeding of $3^{\text {rd }}$ National (1 $1^{\text {st }}$ international conference of biology) (ICBSUM 2021) 5, 6 July

College of Education for Pure Science, University of Mosul, Mosul, Iraq.

غم من مسحوق السيلايت/كغ حنطة اذ كانت النسبة المئوية لقتل اليرقات عندهما 100.0\% وهذا يتطابق مع توصل اليه [25] حيث أشارا إلى أنَّ يرقات خنفساء الخابرا T. granarium لاسيما العمر اليرقي الأول تأثرت بثدة بالمساحيق ولم تكمل حياتها. كما أثرت المساحيق الخاملة على النسبة المئوية لقتل اليرقات وبشكل متفاوت من مسحوق إلى آخر ومن تركيز إلى آخر حيث كانت أعلى نسبة للقتل عند التركيزين 7 و9 غم من مسحوق السيلايت/كغ حنطة اذ كانت نسبة التتل كاملة (100.0\%) مقارنة بالمجوعة الضابطة، أما أقل نسبة قتل بعد المجموعة الضابطة فكانت 20.0\% عند التركيز 5 غم من مسحوق الرمل، وبصورة عامة ارتفعت نسبة القتل بزيادة تركيز المساحيق. أما مدة الطور العذري فانها لم تتأثر معنوياً في اغلب تراكيز المساحيق الخاملة حيث طالت وقصرت بمقدار ضئيل مقارنة بالهجموعة الضابطة وهذا يتفق مع ما ذكره[24] بان التراكيز المستعملة من المساحيق الخاملة لم تؤثر معنويا على مدة الطور العذري،كما أثرت المساحيق الخاملة على النسبة المئوية لقتل العذارى وبشكل طفيف متفاوت من مسحوق إلى آخر ومن تركيز إلى آخر حيث كانت أعلى نسبة للقتل عندالتركيز9 غم من مسحوق الحجر الجيري القيري/كغم حنطة اذ كانت نسبة القتل 38.89\% مقارنة بالمجوعة الضابطة (0.0\%) أما أقل نسبة قتل بعد المجموعة الضابطة فكانت 5.53\% عند التركيز 9 غم من مسحوق الرمل، وكانت هناك فروقات معنويةفي عدد الحشرات الخارجة من طور العذراء من اصل 10 يرقات بين المجموعة الضابطة (10 حشرات كاملة) وجميع تراكيز المساحيق فكان أقل عدد للحشرات الخارجة 0.0 حشرة عند التزكيزين 7 و9 غم من مسحوق السيلايت/ كغم حنطة تلاه التركيز 9 غم من مسحوق الحجر الجيري القيري/ كغم حنطة حيث كان عدد الحشرات الخارجة 1.67 حشرة، أما أعلى عدد للحشرات الخارجة فكان 8.0 حشرة عند التركيز 5 غم من مسحوق الرمل/ كغم حنطة ويعود سبب الاختلاف في عدد الحشرات الكاملة الخارجة بين المساحيق الخاملة وانخفاضها مقارنة بالمجموعة الضابطة إلى ما ذكره [26] بان انخفاض عدد الحشرات الخارجة كان في الغالب بسبب قتل المساحيق لليرقات لاسيما العمر اليرقي الأول.كما كانت هناك فروقات معنويـة في عمر الحشرات البالغة بين المجموعة الضابطة وتراكيز المساحيق وكان اقصر عمر للحشرات البالغة 4.64و 4.67 يوم عند التركيزين7 و9 غم من مسحوق الحجر الجيري القيري/ كغم حنطة، على التوالي، اما اطول عمر للحشرات البالغة فكان 12.17 يوم في المجموعة الضابطة تلاه 7.67 يوم عند التركيز 7 غم من مسحوق الرمل وهذا يتفق مع ما ذكره [24] من أن عمر الحشرة الكاملة لخنفساء الخابرا Tranarium تأثر بثكل معنوي بالمساحيق الخاملة وأنخفض إلى النصف تقريبا لجميع المساحيق مقارنة بمعاملة المقارنة كما ان عمر الحشرة الكاملة انخفض مع زيادة التركيز المستعمل، كما كانت هناك فروقات معنوية في عدد البيض الموضوع / انثى بين المجموعة الضابطة وتراكيز المساحيق حيث كان أعلى عدد للبيض 34.67 بيضة/ انثى في المجموعة الضابطة تلاه 22.0 و 21.33 بيضة/ انثى عند التركيزين 7 و5م من بن مسحوق الرمل/ كغم حنطة، على التوالي، بينما كان اقل عدد للبيض 13.67 بيضة/ انثى عند التركيز 9 غم من مسحوق الحجر الجيري الطباشيري/ كغم حنطة والتركيز 7 غم من مسحوق الحجر الجيري القيري/ كغ من الحنطة، على التوالي، وهذا يشابه ما وجده[27 من ان إناث كل من سوسة الرز Sitophilusoryzae وسوسة الحبوب Sitophilusgranarius المعرضة لحبوب حنطة معاملة بالمساحيق الخاملة مثل الدايتومات الارضية والكاؤلين والكايتولوسس والطين الابيض تضع بيضا أقل من الاناث غير المعرضة للمساحيق الخاملة واوضحا بان سبب ذلك يعود إلى ان التعرض للمساحيق يقصر عمر الحشرة الكاملة وبالتالي يقلل من عدد البيض الموضوع. بينما لم تختلف مدة حضانة البيض معنويـة بين المجموعة الضـابطة وتراكيز المساحيق ماعدا التركيز 5 غم من مسحوق الرمل/ كغم حنطة 
Journal of Education and Science (ISSN 1812-125X), Vol: 30, No: 3, 2021 (167-177)

Special Issue for Proceeding of $3^{\text {rd }}$ National (1st international conference of biology) (ICBSUM 2021) 5, 6 July College of Education for Pure Science, University of Mosul, Mosul, Iraq.

والتركيزين 79 و 9 من مسحوق الحجر الجيري القيري/كغم حنطة وكان الاختلاف (الاطالة والقصر) في مدة حضانة البيض قليلة لا تتجاوز 0.42 يوم. وكانت هناك فروقات معنوية في النسبة المئوية لفقس البيض بين المجموعة الضابطة وتراكيز المساحيق حيث كانت اعلى نسبة مئوية لفقس البيض في المجموعة الضابطة حيث بلغت 85.6\% تلتها 54.73\% عند التركيز 5 غم من مسحوق الرمل/ كغم حنطة بينما كانت أدنى نسبة مئوية لفقس البيض عند التركيز 5 غم مسحوق السيلايت/ كغ حنطة حيث بلغت 15.13\%. جدول 1. تأثير أربعة مساحيق خاملة وبتراكيز مختلفة في حياتية خنفساء الخابرا Trogoderma granarium.

\begin{tabular}{|c|c|c|c|c|c|c|c|c|c|c|}
\hline \multicolumn{9}{|c|}{ معدل الصفات المدروسة } & \multirow{2}{*}{ غم/كغم التركيز } & \multirow[t]{4}{*}{ المساحيق } \\
\hline \% فقس & مدة حضانة & عدد البيض/ & عمر الحشرة & & \%العذارى & مدة الطور & \% اليرقات & مدة الطور & & \\
\hline البيض & البيض & أنثى & الكاملة & الحشرات & الميتة الميتة & العذري & الميتة & اليرقي (يوم) & & \\
\hline & (يوم) (يو) & & & الخارجة & & (يوم) (يو) & & & & \\
\hline cd 36.47 & ab 4.33 & d 18.33 & bc 7.44 & bc 6.0 & ef 9.52 & ab 4.77 & gh 33.3 & *de 27.3 & 5 & الحجر \\
\hline gh 18.90 & ab 4.57 & d 17.67 & d 6.22 & cde 4.33 & cd 18.87 & bc 4.48 & ef 46.7 & c 29.9 & 7 & الجيري \\
\hline efg 26.93 & ab 4.62 & f 13.67 & e 4.97 & def 3.33 & d 16.67 & abc 4.50 & cd 60.0 & ab 32.9 & 9 & الطباشيري \\
\hline C 27.43 & A 4.51 & C 16.56 & C 6.21 & BC 4.56 & B 15.02 & A 4.58 & B 46.67 & BC 30.05 & 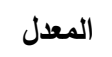 & \\
\hline def 28.90 & ab 4.58 & c 19.67 & d 5.69 & cd 5.33 & e 11.11 & abc 4.56 & fg 40.0 & cd 28.5 & 5 & الحجر \\
\hline fg 24.53 & a 4.74 & f 13.67 & e 4.64 & def 3.67 & c 21.67 & abc 4.64 & de 53.3 & b 32.3 & 7 & الجيري \\
\hline fgh 22.90 & a 4.83 & e 16.0 & e 4.67 & f 1.67 & a 38.89 & c 4.39 & b 73.3 & a 34.2 & 9 & القيري \\
\hline C 25.44 & A 4.72 & C 16.44 & D 4.99 & C 3.56 & AB 23.89 & A 4.53 & B 55.56 & AB 31.69 & 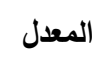 & \\
\hline b 54.73 & b 4.12 & b 21.33 & c 7.04 & b 8.0 & g 0.0 & abc 4.57 & i 20.0 & ef 25.7 & 5 & 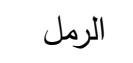 \\
\hline c 42.43 & ab 4.67 & b 22.0 & b 7.67 & $\operatorname{cd} 5.3$ & f 5.56 & a 4.78 & hi 26.7 & de 27.1 & 7 & \\
\hline de 34.57 & ab 4.50 & c 19.33 & c 6.83 & bc 6.0 & f 5.53 & abc 4.63 & g 36.7 & c 29.4 & 9 & \\
\hline В 43.91 & A 4.43 & В 20.89 & B 7.18 & B 6.44 & C 3.69 & A 4.66 & C 27.78 & C 27.41 & المعدل & \\
\hline h 15.13 & ab 4.61 & d 17.67 & d 5.89 & ef 2.67 & b 27.77 & abc 4.60 & c 63.3 & ab 33.8 & 5 & السيلايت \\
\hline-- & -- & -- & -- & -- & -- & **- & a 100 & $* *-$ & 7 & \\
\hline-- & -- & -- & -- & -- & -- & **- & a 100 & $* *-$ & 9 & \\
\hline D 15.13 & A 4.61 & C 17.67 & CD 5.89 & C 2.67 & A 27.77 & A 4.60 & A 87.78 & A 33.85 & المعدل & \\
\hline a 85.60 & ab 4.41 & a 34.67 & a 12.17 & a 10.0 & g 0.0 & ab 4.73 & j 0.0 & f 24.07 & & المجموعة \\
\hline A 85.60 & A 4.41 & A 34.67 & A 12.17 & A 10.0 & C 0.0 & A 4.73 & D 0.0 & D 24.07 & المعدل & الضابطة \\
\hline
\end{tabular}

* الأرقام المتبوعة بأحرف صغيرة وبأحرف كبيرة عمودية متشابهة لا تختلف معنويا حسب اختبار دنكن متعدد الحدود عند مستوى احتمال 0.05.

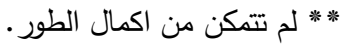


Journal of Education and Science (ISSN 1812-125X), Vol: 30, No: 3, 2021 (167-177)

Special Issue for Proceeding of $3^{\text {rd }}$ National (1 $1^{\text {st }}$ international conference of biology) (ICBSUM 2021) 5, 6 July College of Education for Pure Science, University of Mosul, Mosul, Iraq.

وتبين النتائج في جدول (2) عدم وجود فروقات معنوية في نسب انبات بذور الحنطة المعاملة بتراكيز مختلفة من المساحيق بعد 6 اشهر من المعاملة بين تراكيز المساحيق الخاملة فيما بينها وبين تراكيز المساحيق الخاملة جميعها ومعاملة المجموعة الضابطة وكذلك عدم وجود فروقات معنوية في معدل نسب الانبات بين المساحيق الخاملة والمجموعة الضابطة حيث لم يكن لنوع المسحوق وتركيزه تاثيراً على نسبة الانبات. كانت اعلى معدل نسبة انبات في مسحوق الرمل اذ كانت 86.29\% بينما كانت اقل نسبة انبات في مسحوق الحجر الجيري القيري اذ كانت 83.70\%، كما كانت اعلى نسبة انبات بالنسبة للتراكيز في تركيز 5غم من مسحوق الرمل/ كغ حنطة اذ بلغت 88.9\% بينما اقل نسبة انبات كانت في التركيز7 7 غم من السيلايت/ كغ حنطة اذ بلغت 82.2\%. اتفقت نتائجنا مع ما توصل اليه [24] من ان تخزين بذور الحنطة المعاملة مع المساحيق الخاملة لمدة 6 اشهر لم تتاثر نسبة انباتها معنوياً، كما اتفقت نتائجنا مع اثار اليه[28] من أنّ معاملة بذور اللوبيا بمادة السيلكاجل-60 وبالتركيز 2غم/ كغم بهم لم تؤثر على النسبة المئوية لانبات بذور اللوبيا واكد ذلك[29] اذ ذكروا بان معاملة بذور اللوبيا بمسحوق الطين الخامل لم يؤثر على حيوية البذور عند تخزينها لفترة تزيد عن سنة تحت ظروف التخزين الطبيعية كما تتقق نتائجنا مع ما اشار اليه[25] من ان خلط بذور الحنطة بمسحوق النينفايت لم يظهر تأثيرا معنوياً في نسبة الانبات حتى بعد فترة شهر من التخزين. جدول 2. تأثير المساحيق الخاملة على النسبة المئوية لإنبات بذور الحنطة.

\begin{tabular}{|c|c|c|c|}
\hline المعدل & نسبة الإنبات\% & التركيز غم/كغ & المسحوق \\
\hline \multirow[t]{3}{*}{ A 84.81} & $a * 84.4$ & 5 & الحجر الجيري \\
\hline & a 86.7 & 7 & الطباشيري \\
\hline & a 83.3 & 9 & \\
\hline \multirow[t]{3}{*}{ A 83.70} & a 83.3 & 5 & الحجر الجيري \\
\hline & a 84.4 & 7 & القيري \\
\hline & a 83.3 & 9 & \\
\hline \multirow[t]{3}{*}{ A 86.29} & a 88.9 & 5 & الرمل \\
\hline & a 84.4 & 7 & \\
\hline & a 85.6 & 9 & \\
\hline \multirow[t]{3}{*}{ A 85.19} & a 85.6 & 5 & السيلايت \\
\hline & a 82.2 & 7 & \\
\hline & a 87.8 & 9 & \\
\hline A 85.56 & a 85.6 & & المجموعة الضابطة \\
\hline
\end{tabular}


Journal of Education and Science (ISSN 1812-125X), Vol: 30, No: 3, 2021 (167-177)

Special Issue for Proceeding of $3^{\text {rd }}$ National (1st international conference of biology) (ICBSUM 2021) 5, 6 July

College of Education for Pure Science, University of Mosul, Mosul, Iraq.

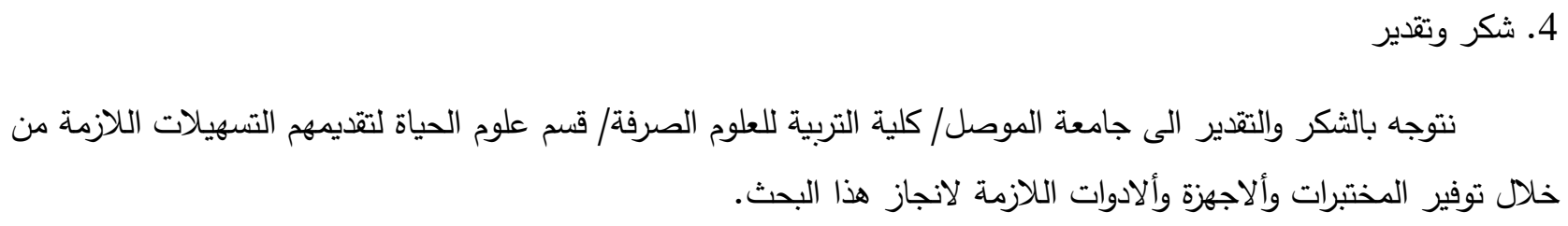

\section{References}

[1] Mir, R. R., Kumar, S., and Shafi, S.(2021). Genetic Dissection for Yield and Yield-Related Traits in Bread Wheat (Triticumastivum L.). In physiological, Molecular, and Genetic Perspectives of Wheat Improvement (Pp.209-227). Spring cham

[2] CIMMYT (2005). CIMMYT Business plan 2006-2010. Translating the vision of seeds of Innovation into a vibrant Work plan. Mexico: Centro Internacional de Mejoramiento de MaizyTrigo.

[3] Pomeranz, Y. (2001). Cereal legume. American Assoc. of cereal chemist, St Peal, MN.PP.821.

[4] Awaida, M., Muhammad, N., Mohmoud, H., and Abdullah, A. (2016). Measuring Analysis of the Economic Efficiency of Wheat crop in the Republic of Iraq. Journal of Agricultural Economic and Social Sciences. 7(6):673-680.

[5] Fiellet, P.(2000). Le grain de ble. Composition et utilisation. Mieuxcomprendre. INRA. P23-24.

[6] Jameel, M. A., Al-Iraqi, R. A., Hussein, A. S. (2011). Susceptibility of red flour beetle to some inert dusts. College of Basic Education Researches Journal, (2): 497-508.

[7] Al-hadidi, S. N., Khamas, N. A., Mtunai, H. A. (2014). Effect of using some spices to control the adults of red flour beetle Triboliumcastaneum (Herbest)(Coleoptera: Tenebrioidae ). Diyala Agricultural Sciences Journal , 6(2): 248-257.

[8] Mohammed, F. K., Alrubeai, H. F., and Khudhair, M. W. (2010). Efficacy of wheat husks and its extracts in protection of stored seeds from infestion by TrogodermagranariumEverts (Coleoptera: Dermestidae),Journal of the college of basic education, 2: 352-358.

[9] Adugna, H. (2006). On-farm storage studies on Sorghum and chickpea in Eritrea. African J. Biotech. 5(17):1537-1544. 
Journal of Education and Science (ISSN 1812-125X), Vol: 30, No: 3, 2021 (167-177)

Special Issue for Proceeding of $3^{\text {rd }}$ National (1 ${ }^{\text {st }}$ international conference of biology) (ICBSUM 2021) 5, 6 July

College of Education for Pure Science, University of Mosul, Mosul, Iraq.

[10] Mehram, E. M., Lotfy. L. M., and Elhassaneen, Y. A. (2019). Infestation of wheat flour by flour beetles (Triboliumcastaneum) affected in its chemical and toxicological properties and validity for human consumption. Journal of Research in the Fields of Specific Education, 22(5): 185201.

[11] Scheff, D. S., Arthur, F. H., Myers, S. W. and Domingue, M. J. (2020) Efficacy Determination of Commercial Deltamethrin-Treated Storage Bags on TrogodermagranariumEverts Adults and Larvae, Agronomy, 10(6):814-822.

[12] Mohan. S., Pretheep-Kumar, P., and Balasubramarian, P.(2010). Insecticide resistance-storedproduct insects. Dusseldorf, LAP Lambert Academic Publishing.

[13] Kavallieratos, N. G., Boukouvala, M. C. Ntalli, N., Skourti, A., karagiannt, E. S., Nika, E. P.,... and Benelli, G. (2020). Effectiveness of eight essential oils against two key storedproduct beetle, prostephanustruncatus (Horn) and TrogodermagranariumEverts.food and chemical toxicology,139-111255.

[14] Kpoviessi, D. A., Chougourou, D.C, Bokononganta, A. H., Fassinou-Hotegni, N. V., and Dossou, J. (2017). Bioefficacy of powdery formulations based on kaolin Powder cashew (Anacardiumoccidentale L.) balms to control Callosobruchusmaculatus F. (Coleoptera, Chrysomelideae: Bruchidae) in stored Cowpea (Vignaunguiculata L.). International Journal of Biological and chemical Sciences.11(4):1424-1436.

[15] Vojoudi, S., Esmaili, M., Farrokhi, M., and Saber, M. (2014). Acute Toxicity of kaolin and essential oils from Menthapulegium and Zingiberofficinale against different stages of Callosobruchusmaculatus under laboratory conditions. Archives of Phytopathology and plant protection, 47(3): 258-291.

[16] Shah, M. A., and Khan, A. A. (2014).Use of diatomaceous earth for the management of storedproduct pests. International Journal of pest management, 60(2): 100-113.

[17] Rees, D. (2004). Insects of stored products. CSIRO publishing 181.

[18] Khan, H. A. A., Akram, W., Lee, S., Manzoor, S., Ayub, S. R., Rehman, K. U., Maqsood, S. (2018). Monitoring susceptibility to spinosad in three major stored-product insect species from Punjab, Pakistan. Pakistan Journal of Zoology, 50(4): 1355-1360. 
Journal of Education and Science (ISSN 1812-125X), Vol: 30, No: 3, 2021 (167-177)

Special Issue for Proceeding of $3^{\text {rd }}$ National (1 ${ }^{\text {st }}$ international conference of biology) (ICBSUM 2021) 5, 6 July

College of Education for Pure Science, University of Mosul, Mosul, Iraq.

[19] Athanassiou, C.G., Philips, T. W., and Wakil, W. (2019). Biology and control of the khapra beetle, Trogodermagranarium, a major quarantine threat to global food security. Annual review of entomology, 64: 131-148.

[20] ISTA. (1985). International rules for Seed testing: Rules 1985. Seed Science and Technology,13.

[21] Haile, A. (2015). Eco-friendly management of Chickpea storage pest, Callosobruchuschinensis L. (Coleoptera; Bruchidae) under laboratory conditions in Eritrea. Journal of Stored Products and Postharvest Research, 6(8): 66-71.

[22] Wijayaratne, L. K. W., and Fields, P.G. (2010). Effect of methoprene on the heat tolerance and cold tolerance of Triboliumcastaneum (Herbst) (Coleoptera: Tenebrionidae). Journal of Stored Products Research, 46(3): 166-173.

[23] SPSS, I. I. B. M. (2011). IBM SPSS statistics for Windows, version 20.0.: IBM Corp, New York, $440 \mathrm{pp}$.

[24] Mohamad, M. A. (2006). Efficacy of numerous inert dusts against Khaprabettle Trogodermagranarium Everts (Coleotera: Dermestidae). Ph.D. Thesis, College of Science University of Mosul, Iraq. 102pp.

[25] Al-Iraqi, R. A. and Ramadan, N. A. (2004). Ninivite dust as new grain protectants against some stored-products insects. Arab Journal of Plant Protection, 22(1): 41-46.

[26] Desmarchelier, J. M., and Dines, J. C. (1987). Dryacide treatment of stored wheat: its efficacy against insects, and after processing. Australian Journal of Experimental Agriculture, 27(2): 309-312.

[27] El-Nahal, A. K. M., and El-Halfawy, M. A. (1973). The effects of sublethal treatments with pyrethrins and certain inert dusts on some biological aspects of Sitophilusoryzae L. and S. granarius L. (Coleoptera). Bull. Entomol. Soc. Egypt, Econ. Ser, 7:253-260.

[28] Mahdi, M. T. and Hamoudi, R. F. (1987). The effect of some dust and oil protectants on the biology of cowpea beetle, Callosobruchusmaculates F. (Coleoptera: Bruchidae). Arab Journal of Plant Protection, 5:48-52. 
Journal of Education and Science (ISSN 1812-125X), Vol: 30, No: 3, 2021 (167-177)

Special Issue for Proceeding of $3^{\text {rd }}$ National (1 ${ }^{\text {st }}$ international conference of biology) (ICBSUM 2021) 5, 6 July College of Education for Pure Science, University of Mosul, Mosul, Iraq.

[29] Babu, T. R., Hussaini, S. H., and Satyanarayana, B. (1989). Effect of pre-storage seed treatments on adult mortality, oviposition and development of Callosobruchuschinensis L. (Bruchidae: Coleoptera), and the viability of mungbean (Vignaradiata (L.) Wilczek) in India. International Journal of Pest Management. 35(4): 397-398. 